\title{
Dendritic Transport: Quantitative Analysis of the Time Course of Somatodendritic Transport of Recently Synthesized RNA
}

\author{
Lauren Davis, ${ }^{a}$ Barbara Burger, Gary A. Banker, and Oswald Steward \\ Departments of Neuroscience and Neurosurgery and the Neuroscience Program, University of Virginia, School of \\ Medicine, Charlottesville, Virginia 22908
}

\begin{abstract}
We have previously reported that recently synthesized RNA is selectively transported into the dendrites of hippocampal neurons grown in culture (Davis et al., 1987). The present study provides further details about this transport process, focusing especially on the velocity of transport, by comparing the velocity of dendritic transport of RNA in neurons of different ages and in the branched and unbranched dendrites of individual neurons. In our previous study, we recognized that calculations of transport velocity could be compromised because transport was being evaluated in a population of dendrites of varying lengths. The present study uses a mathematical modeling approach to determine how the morphology of the population of dendrites would affect the analysis of transport velocity. Focusing first on a simple model, we compared the distribution of transported material at various times when all dendrites were of the same length and when the population included dendrites of different lengths. We found that the distance of labeling increased linearly over time when all dendrites were of the same length, but increased with a negatively accelerating curve when dendrites were of different lengths. We then determined the actual distribution of dendritic lengths in cultured hippocampal neurons, based on immunostaining with an antibody directed against the selective dendritic marker, microtubuleassociated protein 2 (MAP2). Using a computer model, we calculated the mean distance of transport as a function of time in this population of dendrites, assuming different velocities of transport. The velocity that best fit the measured distances of RNA transport in both 7- and 15-d-old neurons was $11 \mu \mathrm{m} / \mathrm{hr}(0.26 \mathrm{~mm} / \mathrm{d})$. However, for the dendrites exhibiting the longest distance of labeling, the best-fitting curve assumed a velocity of $21 \mu \mathrm{m} / \mathrm{hr}$ in both 7- and 15-d-old neurons $(0.50 \mathrm{~mm} / \mathrm{d})$. Comparisons of transport in branched and unbranched dendrites revealed that the distance of labeling over branched dendrites was consistently longer than
\end{abstract}

\footnotetext{
Received Mar. 19 1990; revised May 4, [890; accepted May 8, 1990.

We wish to thank Dr. H. Kutchai for helpful discussions about intracellular transport. We also wish to thank T. M. Breeden for excellent training and support in using the Gould IP8500, Dr. W. B. Levy for the use of the Bioquant image analysis system, and Dr. P. C. Brunjes for the use of the Sigma Scan image analysis system. A version of this paper was included in a dissertation submitted by $L$. Davis in partial fulfillment of the requirements for the degree of Doctor of Philosophy, the Neuroscience Program, University of Virginia. This work was supported by NIH Grant NS23094 to G.B. and O.S. L.D. was the recipient of a predoctoral fellowship from NIH Institutional Training Grant NS07199.

Correspondence should be addressed to Oswald Steward, Ph.D., Department of Neuroscience, University of Virginia, School of Medicine, Box 230, Charlottesville, VA 22908.

Present address: Department of Anatomy and Neurobiology, Colorado State University, Fort Collins, CO 80523.

Copyright (c) 1990 Society for Neuroscience $0270-6474 / 90 / 093056-13 \$ 03.00 / 0$
}

over unbranched dendrites of individual neurons. However, neurons with a larger proportion of branched dendrites did not exhibit a greater mean distance of transport. The density of silver grains was higher over branched than over unbranched dendrites, suggesting that a greater amount of recently synthesized RNA may be transported into branched dendrites. Taken together, these results suggest that RNA transport into dendrites is regulated differentially in the dendrites of individual neurons.

In a previous study, we described and partially characterized a transport system that delivers recently synthesized RNA into the dendrites of hippocampal neurons in culture (Davis et al., 1987). Following pulse labeling with ${ }^{3} \mathrm{H}$-uridine, we found that recently synthesized RNA was initially restricted to the nucleus; subsequently, the material was transported selectively into dendrites. This dendritic transport was blocked by metabolic poisons, and the RNA in transit appeared to be bound to the cytoskeleton, because much of it remained following detergent extraction of the cells. Other studies have shown that proteinsynthetic machinery, comprised of polyribosomes and membranous cisterns, is present in dendrites and is selectively localized beneath synaptic sites (Steward and Levy, 1982). Polyribosomes are particularly prominent beneath developing synapses (Steward and Falk, 1986) and when synapses are being replaced following denervation (Steward, 1983). We have proposed that the transport mechanism that we have characterized may be responsible for delivering this protein-synthetic machinery into dendrites and that there may be a mechanism for targeting particular messages to particular locations (Steward et al., 1988; see also Garner et al., 1988; Gordon-Weeks, 1988; Papandrikopoulou et al., 1989; Tucker et al., 1989).

The present study provides further details about the dendritic transport of RNA, focusing especially on the velocity of transport. In our previous study, we estimated the velocity of transport by measuring the average distance of labeling in all the dendrites at various times after the pulse. Measurements of the increases in the distance of labeling as a function of time in the total population of dendrites suggested a velocity of transport of about $0.20-0.25 \mathrm{~mm} / \mathrm{d}$. It was clear, however, that such calculations were problematic because the velocity of transport can be accurately determined from the mean distance of labeling only when labeling has not reached the tips of any dendrites. Because some of the dendrites of hippocampal neurons are quite short, labeled material would reach their tips quickly. Inclusion of these short dendrites in the overall averages would lead to an underestimate of the velocity of transport, particularly at the longer intervals. 
In an attempt to circumvent this problem, we also measured the time course of the increases in labeling for the single dendrite per neuron that exhibited the longest distance of labeling, on the assumption that this might provide a more accurate estimate of the actual velocity of transport. The apparent velocity of transport in the dendrites with the longest distance of labeling was $0.4-0.5 \mathrm{~mm} / \mathrm{d}$, which we proposed as the best estimate of the actual velocity of transport.

The above conclusion is based upon the assumption that measurements of the velocity of transport in the total population substantially underestimate the actual velocity because of the inclusion of short dendrites in the sample. If this is true, a better estimate of velocity would be obtained by concentrating on the longest dendrites. Another possibility, however, is that the velocity of transport is actually faster in longer dendrites. One of the principal goals of the present study is to distinguish between these possibilities.

The present study uses a mathematical modeling approach to evaluate more directly how measures of the velocity of transport would be affected by the distribution of dendritic lengths in the population of cells being analyzed. The model calculates the expected mean distance of labeling as a function of time, in a given population of dendrites, and assuming a given velocity of transport.

Using this model, we examined how differences in dendritic length affect the measures of the average distance of transport over time. We then used the actual distribution of dendritic lengths in the cultures to estimate the average velocity of transport in the dendrites of hippocampal neurons, as well as the relationship between transport velocity and dendritic length. Surprisingly, thesc calculations indicate that the observed differences in the distance of transport over time between the longest dendrites and the total population of dendrites are not a measurement artifact, but may reflect real differences in dendritic transport.

The present study also evaluates, RNA transport in dendrites of different ages and morphologies. We examined the velocity of transport in hippocampal neurons after $7 \mathrm{~d}$ in culture, when the dendrites are quite immature, and after $15 \mathrm{~d}$, when they are longer and more extensively branched (Banker and Waxman, 1988). We report here that the velocity of transport is comparable in neurons of different ages but that transport is consistently faster in the longest dendrites at each age.

\section{Materials and Methods}

Preparation of hippocampal cultures. Cultures of hippocampal neurons were prepared as previously described (Bartlett and Banker, 1984a; see also Goslin and Banker, 1990). Neurons obtained from 18-19-d-old fetuses were plated onto polylysine-coated coverslips at a density of $6000 / \mathrm{cm}^{2}$ (glass coverslips in experiments $15 \mathrm{~A}, 15 \mathrm{~B}, 7 \mathrm{~A}$; plastic coverslips in experiment 7B). At this density, the dendritic arborizations of individual neurons are easily visualized (Banker and Cowan, 1979; Bartlett and Banker, 1984a). After the cells had attached (3-4 hr), the coverslips were placed cell side down over a monolayer of glial cells and maintained at $36^{\circ} \mathrm{C}$ in minimum essential medium (MEM) supplemented with the N2 mixture of Bottenstein and Sato (1979). Cytosine arabinoside (final concentration $5 \mu \mathrm{M}$ ) was added $3 \mathrm{~d}$ after plating in 3 experiments (experiments 15B, 7A, 7B). A total of 4 cultures were analyzed, 2 of which were maintained for 7 days in vitro (experiments 7A, 7B), and 2 of which were maintained for $15 \mathrm{~d}$ (experiments 15A, $15 B)$.

Pulse-chase experiments. Experiments were carried out using neurons that had been maintained in culture for 7 or $15 \mathrm{~d}$. For pulse labeling, coverslips were transferred to a petri dish containing $40 \mu \mathrm{Ci} / \mathrm{ml} 5,6-{ }^{3} \mathrm{H}$ uridine (New England Nuclear; specific activity, $39-47 \mathrm{Ci} / \mathrm{mmol}$ ) in glial-conditioned medium. Coverslips were maintained in the incubation medium for $1 \mathrm{hr}$ at $36^{\circ} \mathrm{C}$, then rinsed twice in MEM $\left(36^{\circ} \mathrm{C}\right)$ to remove the precursor and either fixed immediately $(0 \mathrm{hr}$ chase) or returned to the original dishes containing glial-conditioned medium to which an excess of unlabeled uridine $\left(10^{-5} \mathrm{M}\right)$ had been added. Five or 6 coverslips were fixed for autoradiography at chase intervals of 0 , $3,6,9,12$, and $24 \mathrm{hr}$; in addition, coverslips werc fixed at a chase interval of $1.5 \mathrm{hr}$ in experiment $15 \mathrm{~B}, 7 \mathrm{~A}$, and $7 \mathrm{~B}$. Fixation was accomplished by immersing the coverslips in $4 \%$ paraformaldehyde in PBS containing $0.12 \mathrm{M}$ sucrose for $20-40 \mathrm{~min}$ at $36^{\circ} \mathrm{C}(\mathrm{pH}, 7.3)$. The coverslips were rinsed in PBS, dehydrated in graded ethanols, and air dried.

Autoradiography of radiolabeled cultures. Coverslips were mounted cell side up on glass slides with Gurr's Fluoromount and dried flat overnight. The glass slides were dipped in undiluted Kodak NTB-2 emulsion $\left(41^{\circ} \mathrm{C}\right)$, dried for at least $2 \mathrm{hr}$, packed with dessiccant in lighttight boxes, and stored at $4^{\circ} \mathrm{C}$. Autoradiograms were developed in Kodak D-19 $\left(15^{\circ} \mathrm{C}\right)$ for $3 \mathrm{~min}$ and fixed in Kodak Rapid-Fix for $4 \mathrm{~min}$. Slides were coverslipped with Kaiser glycerol jelly ( $8 \%$ gelatin, $0.1 \%$ phenol in 1:1 glycerine and water) and dried overnight.

Determination of exposure interval. Determination of the autoradiographic exposure is critical in experiments such as the present one, in which high concentrations of ${ }^{3} \mathrm{H}-\mathrm{RNA}$ are found in the nucleus and in which transport may be represented by relatively lower concentrations of ${ }^{3} \mathrm{H}-\mathrm{RNA}$ in distal dendrites. The duration of the exposure must be gauged so that low concentrations of ${ }^{3} \mathrm{H}-\mathrm{RNA}$ are above the threshold for detection. Therefore, we sought to define conditions so that the apparent distance of dendritic labeling did not increase with increasing exposure intervals. Fifteen-day-old cultures that had transported ${ }^{3} \mathrm{H}-$ RNA for $9 \mathrm{hr}$ were prepared for autoradiography. We then compared the mean distance of labeling in dendrites after autoradiographic exposure for $2,4,7$ or $14 \mathrm{~d}$. We found that the apparent distance of labeling increased between 2 and $7 \mathrm{~d}$ of autoradiographic exposure but did not increase further between 7 and $14 \mathrm{~d}$. Therefore, for all quantitative analyses, autoradiograms were exposed for 6 or $7 \mathrm{~d}$.

Selection of neurons for quantitation. Fifteen to 25 neurons from each chase interval were selected for the quantitative analyses (see Table 1). Neurons were selected on the basis of predetermined microscope-stage coordinates and photographed in dark-field illumination. Cells were included in the sample only if they (1) exhibited a characteristic neuronal morphology (cell body diameter of 10-20 $\mu \mathrm{m}$, dendrites with characteristic diameter and taper), (2) had heavy labeling over the nucleus and, at chase intervals longer than $0 \mathrm{hr}$, over the cell body, and (3) were separated from the somata of other cells by at least $50 \mu \mathrm{m}$. Dendrites that contacted a labeled portion of another cell or looped around to contact the cell of origin were excluded from the analysis because it was impossible to determine where the labeled dendrite of one cell stopped and the dendrite of another cell began. In all, only a small number of dendrites were excluded on the basis of these criteria $(0-10 \%$ of the total in each group).

Calculation of the distance of labeling. Photomicrographs of all of the neurons were printed at a nominal magnification of $475 \times$, and the distance of labeling in every dendrite was measured directly from the photomicrographs. In experiments $15 \mathrm{~B}, 7 \mathrm{~A}$, and $7 \mathrm{~B}$, the chase interval of each photographed neuron was masked, so scoring was blind. To determine the site at which labeling in a dendrite ended, we prepared a template circle that was $10 \mu \mathrm{m}$ in diameter relative to the photographic magnification. The end of labeling in each dendrite was taken as the most distal point where (1) at least 3 silver grains were present in the field inscribed by the template circle and (2) where all silver grains proximal to that point were no more than $10 \mu \mathrm{m}$ apart. Occasionally, dendrites were sparsely labeled for a short length (gaps 10-30 $\mu \mathrm{m}$ between adjacent silver grains), but the labeling increased distal to the gap. In these instances, if there was more than 1 template circle with 3 silver grains distal to the gap, then the end of labeling was marked at the heavier label distal to the gap.

The distance of labeling was determined by measuring the distance from the center of the nucleus to the end of labeling in each dendrite using a Bioquant image analysis system (R\&M Biometrics, Inc.). The mean distance of labeling in the total population of dendrites at each chase interval was calculated. In addition, we identified the single dendrite per neuron in which labeling extended farthest, and the mean distance of labeling in these dendrites was also calculated $(N=25$ dendrites at each chase interval).

Calculation of the distance of labeling in branched and unbranched dendrites. The mean distance of labeling in branched and unbranched 
dendrites was calculated at chase intervals of 1.5 and $3 \mathrm{hr}$ (7-d-old cultures) or 1.5-6 hr (15-d-old cultures). Dendrites were classified directly from the photographs as branched or unbranched. In some cases, it was difficult to determine whether 2 dendrites extended from approximately the same location on the soma or whether a single dendrite branched at, or very close to, the soma. Therefore dendrites were considered branched only if the branching occurred $20 \mu \mathrm{m}$ or more from the center of the nucleus. Dendrites shorter than $20 \mu \mathrm{m}$ were excluded from the analysis. The distance of labeling in branched dendrites was calculated as the distance of labeling in the branchlet with the longest distance of labeling.

Calculation of the density of silver grains in branched and unbranched dendrites. The density of silver grains in all the dendrites of 4 neurons at chase intervals of 3,6 , and $12 \mathrm{hr}$ in experiment $15 \mathrm{~A}$ was measured with the aid of a Gould IP8500 Image Analysis System and software developed by the University of Virginia Biomedical Image Processing Center. Neurons were viewed in bright-field illumination using a Leitz Orthoplan microscope, and the microscopic image was recorded by a Dage-MTI 68 Newvicon camera and digitized. Grains were identified, and grain density was evaluated in a circular counting window $7 \mu \mathrm{m}$ in diameter. Statistical comparisons of grain density were carried out at distances of $25,50,75,100,125,150$, and $175 \mu \mathrm{m}$ from the center of the nucleus.

Measurement of the length of dendrites. To determine the length of the dendrites, we took advantage of the fact that microtubule-associated protein 2 (MAP2) is selectively localized in dendrites and somata (Matus et al., 1981; Caceres et al., 1984b). The MAP2 antibody used, AP-14, is a mouse monoclonal antibody and was a gift from L. I. Binder (Binder et al., 1986). For immunocy tochemistry, sister cultures of those prepared for autoradiography were fixed as described above, permeabilized with $100 \%$ ethanol for $30 \mathrm{~min}$, rehydrated in graded ethanols, and rinsed with PBS. Cultures were prcincubated with $3 \%$ normal rabbit serum for $30 \mathrm{~min}$ at $37^{\circ} \mathrm{C}$, then incubated overnight at $4^{\circ} \mathrm{C}$ with $\mathrm{AP}-14$ at a dilution of $1: 400$ at $4^{\circ} \mathrm{C}$. After washing for $15 \mathrm{~min}$ with PBS, coverslips were incubated in rabbit anti-mouse IgG $(1: 40)$ for $2 \mathrm{hr}$ at $37^{\circ} \mathrm{C}$, followed by peroxidase antiperoxidase $(1: 80)$ for $30-60 \mathrm{~min}$. They were then reacted with diaminobenzidine as previously described (Caceres et al., 1986).

MAP2-stained neurons were selected on the basis of predetermined microscope-stage coordinates and photographed in dark-field illumination as above. When dendrites grew close to each other, the cultures were examined at a high magnification to determine with certainty where each dendrite ended. The lengths of the dendrites were measured using a Bioquant image analysis system (R\&M Biometrics, Inc.). The average length of the total population of dendrites and the longest dendrite of each neuron was calculated as described above. The distance between the center of the nucleus and the most proximal branchpoint of each branched dendrite was measured using a Sigma Scan image analysis system.

In our previous report (Davis et al., 1987; data from experiment 15A), dendritic lengths were measured from bright-field photomicrographs. After additional analyses, we concluded that peroxidase reaction product could be more accurately determined using dark-field illumination and we have used that approach for the measurements reported here. In experiment $15 \mathrm{~A}$, there was a small difference in mean dendritic length measured in dark-field as compared with bright-field illumination (bright field, $98 \pm 59$; dark field $90 \pm 51$ ); in experiment $15 \mathrm{~B}$, mean dendritic lengths were identical (bright field, $81 \pm 42$; dark field, $81 \pm 40$ ).

\section{Results}

\section{Qualitative observations}

In a previous study, we briefly described the pattern of labeling in hippocampal neurons that had grown in culture for $15 \mathrm{~d}$ after exposure to ${ }^{3} \mathrm{H}$-uridine (experiment $15 \mathrm{~A}$ of the present report). These findings were confirmed in an additional experiment (15B). Comparable studies of dendritic transport of RNA in 7-d-old cultures were also undertaken (7A, 7B). A description of these observations will be summarized here to provide background for the quantitative analyses.

Neurons prepared for autoradiography at the end of a 1-hr pulse with ${ }^{3} \mathrm{H}$-uridine ( $0 \mathrm{hr}$ chase) exhibited heavy labeling over nuclei (Fig. $1 A$ ). A few silver grains were present over the perikaryon and the most proximal dendritic regions in some neu- rons. By the end of the 1.5 -hr chase interval, perikarya were as heavily labeled as nuclei, and the proximal regions of all dendrites exhibited heavy labeling for a distance of 10-30 $\mu \mathrm{m}$. At chase intervals of $3 \mathrm{hr}$ and longer; the distance of labeling in dendrites increased progressively. At the shorter survival intervals (3-6 hr), when heavy labeling was restricted to proximal segments, light labeling was sometimes observed distal to the leading edge of dense labeling (see Fig. 1). The significance of this labeling, which was below the criterion used for analysis, is uncertain. At intervals less than $12 \mathrm{hr}$, it was our impression that dense labeling extended farther in branched dendrites than in unbranched dendrites of the same neuron (Fig. 1B). By 12 $\mathrm{hr}$, the leading edge of dense labeling appeared to have reached the tips of all dendrites (Fig. $1 C$ ). Labeling appeared to be more sparsely distributed in distal dendrites at the $24-\mathrm{hr}$ chase interval than at $12 \mathrm{hr}$ (Fig. 1D). Often, the silver grains were present in clusters (approximately 4-10 silver grains per cluster; Fig. $1 E$ ). Many of these clusters appeared to lie at the intersection between a dendrite and a presumptive axon (Fig. 1, $E, F$ ).

In general, the pattern of labeling in 7-d-old neurons was similar to that in 15-d-old neurons (data not shown). At the end of the 1-hr pulse, labeling was, for the most part, restricted to the nucleus. Over time, the cell body and then the dendrites became heavily labeled. Labeling appeared to reach the tips of the dendrites by $6 \mathrm{hr}$.

In addition to the dense labeling of dendrites, a few putative axons, identified by their characteristic morphology (Bartlett and Banker, 1984a, b), were lightly labeled in both 7- and 15d-old cultures (see Fig. 2), particularly at intervals of 12 and 24 hr. The axonal labeling was patchy, and frequently, unlabeled scgments more than $20 \mu \mathrm{m}$ long separated the labeled regions. Over the segments of the axons that exhibited labeling, grain density ranged between 1 and 5 silver grains per $20 \mu \mathrm{m}$. This was much lower than the $5-20$ or more grains per $20 \mu \mathrm{m}$ observed over the labeled segments of the dendrites.

Because the axonal labeling was so light, it was impossible to determine whether all axons were labeled or whether the labeling was restricted to a small number of axons. To examine this further, 12-d-old cultures were exposed continuously to ${ }^{3} \mathrm{H}$ uridine $(40 \mu \mathrm{Ci} / \mathrm{ml})$ for 26 or $47 \mathrm{hr}$. In autoradiograms prepared from these cultures, labeling in dendrites was very dense. Under these conditions, most axons were lightly labeled. The axonal labeling may reflect transport of 4S RNA from the cell body (Black and Lasek, 1977; Zanakis et al., 1984), local synthesis of RNA by mitochondria, or some other process.

\section{Quantitative analyses of the transport of ${ }^{3} \mathrm{H}$-RNA into dendrites}

The time course of the increase in the distance of dendritic labeling in 7- and 15-d-old neurons is illustrated in Figure 3. Figure $3 A$ illustrates the average distance of labeling at each chase interval in the total population of dendrites, and Figure $3 B$ illustrates the average distance of labeling in the single dendrite per neuron exhibiting the longest distance of labeling. For the first $3 \mathrm{hr}$, the rate of increase in the distance of labeling appeared comparable in 7-and 15-d-old neurons for hoth the total population and the dendrites with the longest distance of labeling. Thereafter, the rate of increase was greater in the 15d-old neurons.

A Wilcoxon matched-pairs signed-rank test was employed to determine the reproducibility between experiments of each age. Comparisons of the curves illustrating the increases in the mean 

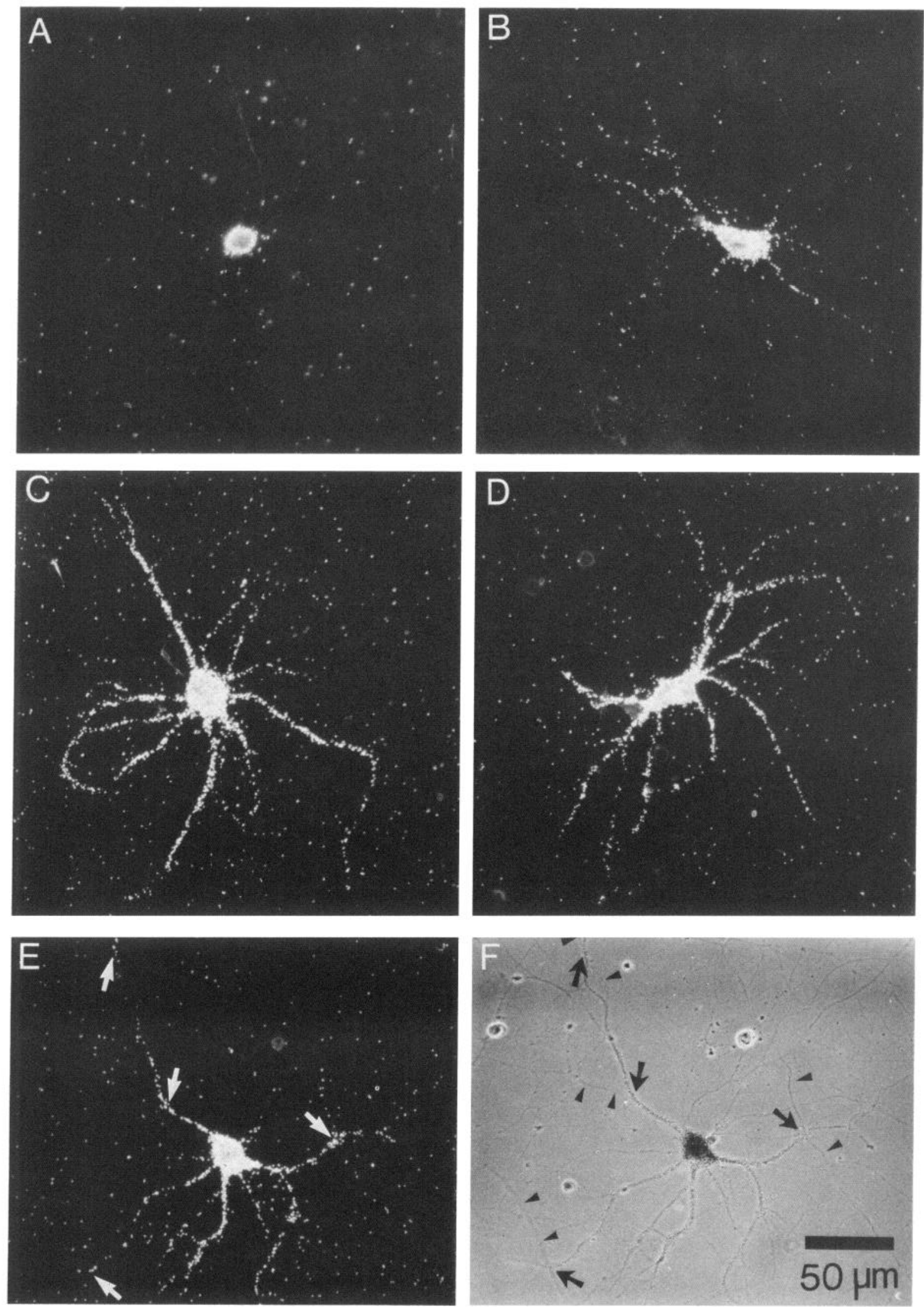

Figure 1. Transport of recently synthesized RNA by neurons in culture. A, Pattern of labeling of a neuron fixed immediately after pulse labeling with ${ }^{3} \mathrm{H}$-uridine for $1 \mathrm{hr}(0 \mathrm{hr}$ chase). $B, C$, and $D$, Pattern of labeling after chase intervals of 3,12 , and $24 \mathrm{hr}$ respectively. $E$ and $F$, Dark-field and phase-contrast photomicrographs, respectively, of a neuron fixed after a 12-hr chase. Note clusters of silver grains at some of the intersections of axons (arrowheads) and dendrites (arrows). 


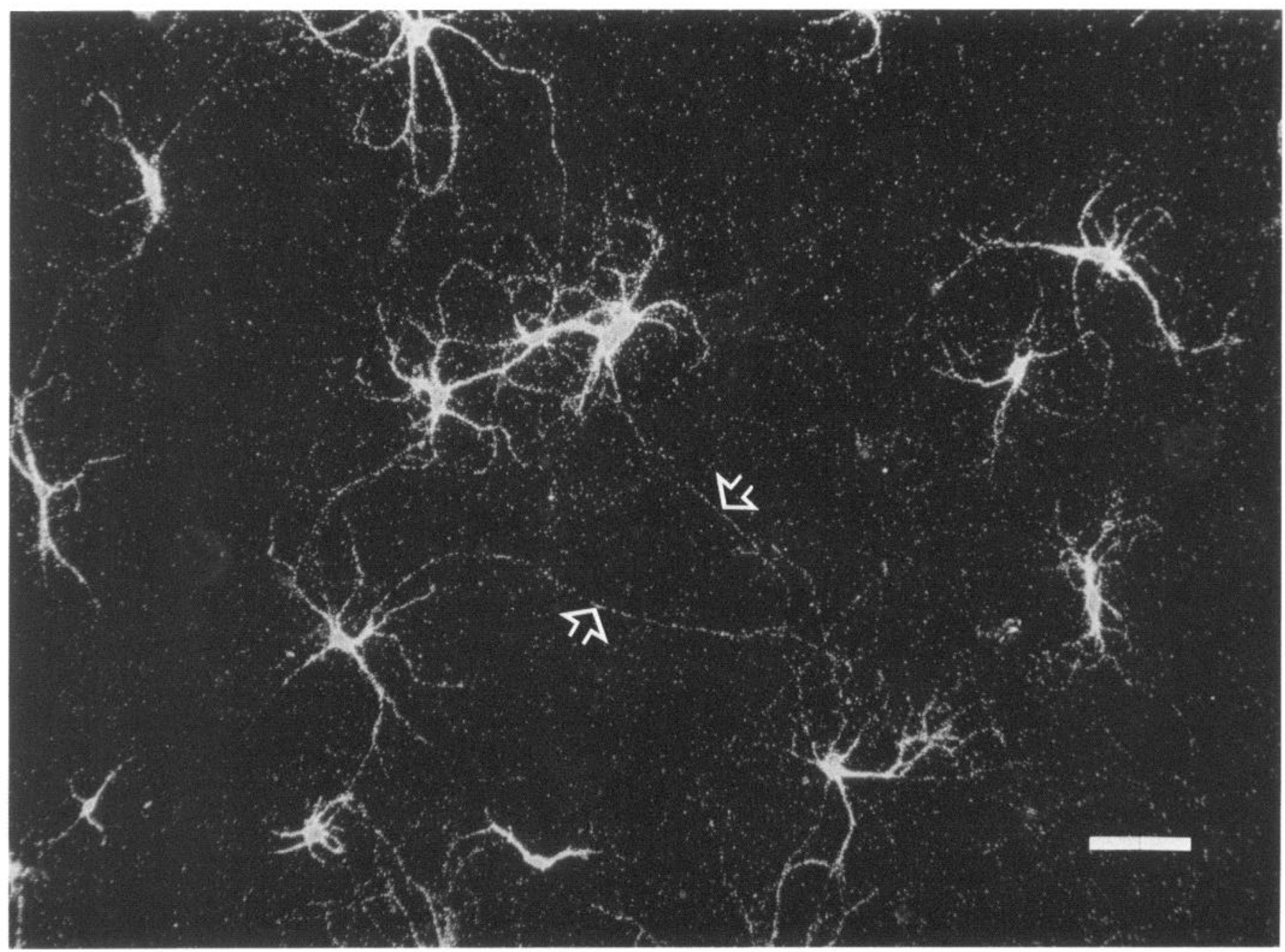

Figure 2. Transport of recently synthesized RNA into some axons. The figure illustrates 2 labeled axons of neurons fixed $24 \mathrm{hr}$ after the 1 -hr pulse of uridine. These are among the most heavily labeled axons found in the cultures. Axons are marked by arrows. Scale bar, $100 \mu \mathrm{m}$.

\section{Table 1. Summary of experiments and data sampling}

\begin{tabular}{|c|c|c|c|c|}
\hline \multirow{2}{*}{$\begin{array}{l}\text { Transport } \\
\text { interval } \\
\text { age }\end{array}$} & \multicolumn{4}{|c|}{ Experiment number } \\
\hline & $\begin{array}{l}15 \mathrm{~A} \\
15 \text { days }\end{array}$ & $\begin{array}{l}15 \mathrm{~B} \\
15 \text { days }\end{array}$ & $\begin{array}{l}7 \mathrm{~A} \\
7 \text { days }\end{array}$ & $\begin{array}{l}7 \mathrm{~B} \\
7 \text { days }\end{array}$ \\
\hline $0 \mathrm{hr}$ & 87 & 124 & 105 & 77 \\
\hline $1.5 \mathrm{hr}$ & $a$ & 188 & 245 & 130 \\
\hline $3 \mathrm{hr}$ & 207 & 264 & 224 & 142 \\
\hline $6 \mathrm{hr}$ & 231 & 328 & 259 & 140 \\
\hline $9 \mathrm{hr}$ & 263 & 312 & 263 & 154 \\
\hline $12 \mathrm{hr}$ & 259 & 393 & 317 & 137 \\
\hline $24 \mathrm{hr}$ & 218 & 317 & 308 & 158 \\
\hline MAP2 & 285 & 372 & 261 & 132 \\
\hline
\end{tabular}

The total number of dendrites analyzed at each chase interval for all 4 experiments is listed. Two of the experiments were begun when the cultures had been growing for $7 \mathrm{~d}$, and 2 experiments were begun at $15 \mathrm{~d}$ in vitro. The number of neurons analyzed at each chase interval was as follows: $15 \mathrm{~A}, 25$ neurons randomly selected from one coverslip prepared at each chase interval; $15 \mathrm{~B}$ and $7 \mathrm{~A}, 5$ neurons randomly selected from each of 5 coverslips prepared at each chase interval; 7B, 5 neurons randomly selected from each of 3 coverslips prepared at each chase interval.

a Cultures were not examined at the $1.5-\mathrm{hr}$ chase interval in experiment $15 \mathrm{~A}$ distance of labeling in the total population of dendrites over time, as well as in the dendrites with the longest distance of labeling, revealed that there were no significant differences between the experiments in 15-d-old cultures $(p>0.05)$. The mean distance of labeling after $12 \mathrm{hr}$ of transport was approximately equal to the mean length of the dendrites in MAP2-stained sister cultures, for both the total population of dendrites and the dendrites with the longest distance of labeling (see Fig. 3, $A, B$ ). The time course of transport in the dendrites exhibiting the longest distance of labeling differed significantly from that in the total population of dendrites (experiment 15A, $p=0.025$; experiment $15 \mathrm{~B}, p=0.01$ ).

The standard errors for each measurement of mean distance of labeling are not illustrated in Figure 3 because the standard deviation of each measurement is large (about $50 \%$ of the mean). The high variability in the mean distance of labeling probably reflects the fact that there is a large variability in the lengths of the dendrites themselves (i.e., the standard deviation of the measurements of the length of MAP2-stained dendrites is also about $50 \%$ of the mean).

Wilcoxon matched-pair signed-rank tests revealed differences 
Table 2. Comparison of the path lengths of MAP2-stained dendrites with the distances of labeling in all dendrites

\begin{tabular}{lllll}
\multirow{2}{*}{$\begin{array}{l}\text { Trans- } \\
\text { port } \\
\text { interval }\end{array}$} & \multicolumn{4}{l}{ Days in culture } \\
\cline { 2 - 5 } & $7 \mathrm{~A}$ days & $7 \mathrm{~B}$ & $15 \mathrm{~A}$ days & $15 \mathrm{~B} 2$ \\
\hline $0 \mathrm{hr}$ & $17.5 \pm 6^{* *}$ & $18.7 \pm 5^{* *}$ & $23.0 \pm 10^{* *}$ & $21.2 \pm 10^{* *}$ \\
$1.5 \mathrm{hr}$ & $30.6 \pm 16^{* *}$ & $28.8 \pm 10^{* *}$ & & $27.0 \pm 12^{* *}$ \\
$3 \mathrm{hr}$ & $38.8 \pm 24^{* *}$ & $45.5 \pm 20^{*}$ & $46.7 \pm 26^{* *}$ & $56.9 \pm 28^{* *}$ \\
$6 \mathrm{hr}$ & $43.2 \pm 26^{*}$ & $55.3 \pm 28$ & $60.9 \pm 35^{* *}$ & $59.4 \pm 33^{* *}$ \\
$9 \mathrm{hr}$ & $46.6 \pm 25$ & $47.6 \pm 23^{*}$ & $74.8 \pm 44^{*}$ & $55.5 \pm 36$ \\
$12 \mathrm{hr}$ & $43.3 \pm 26^{*}$ & $51.9 \pm 26^{*}$ & $85.3 \pm 47$ & $81.6 \pm 43$ \\
$24 \mathrm{hr}$ & $53.3 \pm 34$ & $63.2 \pm 31^{*}$ & $78.5 \pm 51^{*}$ & $88.5 \pm 47$ \\
MAP2 & $50.1 \pm 28$ & $56.1 \pm 28$ & $90.4 \pm 51$ & $80.8 \pm 39$
\end{tabular}

The mean distance of labeling at each transport interval is listed for each experiment. The mean dendritic length in MAP2-stained sister cultures is listed on the bottom line. Distance of labeling and length are given in $\mu \mathrm{m} \pm \mathrm{SD}$. Analyses comparing the distances of labeling with the dendritic lengths employed tests of least-square mean ranks ( $t$ statistics)

$* p<0.05$.

${ }^{* *} p<0.0001$

between the 2 experiments involving 7-d-old neurons. The 2 curves illustrating the time course of the increases in the distance of labeling in the total population of dendrites differed significantly ( $p \leq 0.025$ ). These differences may be due to slight differences in dendritic lengths between different neuronal cultures. There was no significant difference between the 2 experiments in the time course of transport in the dendrites with the longest distance of labeling (Wilcoxon; $p>0.05$ ). As in 15-d-old cells, the time course of the increases in the distance of labeling was significantly faster in the dendrites exhibiting the longest distance of labeling than in the total population of dendrites (Wilcoxon; $p=0.01$ for both experiments).

The time course of transport differed at different ages, for both the total population of dendrites and the dendrites with the longest distance of labeling $(p \leq 0.025)$. Tests of least-square mean ranks revealed that there were no significant differences between any experiments at the 0 - or 1.5-hr chase interval ( $p$ $>0.05)$, but there were significant differences $(p<0.05)$ at all the other intervals (3-24 hr). Thus, the time course of transport in 7-and 15-d-old cells is similar at early time intervals. The differences at later time intervals presumably reflects the shorter lengths of the dendrites of 7-d-old neurons (see below).

The graphs of Figure 3 suggest that label reaches the tips of the dendrites of 7-d-old neurons within about $6 \mathrm{hr}$ but does not reach the tips of the dendrites of 15-d-old neurons until about $12 \mathrm{hr}$ (see Fig. 3). Statistical comparisons of the distance of labeling with the average length of the dendrites (as revealed by MAP2 staining) confirm this conclusion. Table 2 compares the distances of labeling at each chase interval with the dendritic lengths in sister cultures that had been stained with MAP2. In 15-d-old neurons, the mean distance of labeling at all early chase intervals was significantly less than the mean dendritic length (at least $p<0.0001$ ). By $12 \mathrm{hr}$, there was no significant difference between dendritic length and the distance of labeling in either experiment (see Table 2), suggesting that recently synthesized RNA had become distributed throughout the dendritic arbor. In 7-d-old neurons, the average distance of labeling at $0,1.5$, and $3 \mathrm{hr}$ differed significantly from the average dendritic length.
A
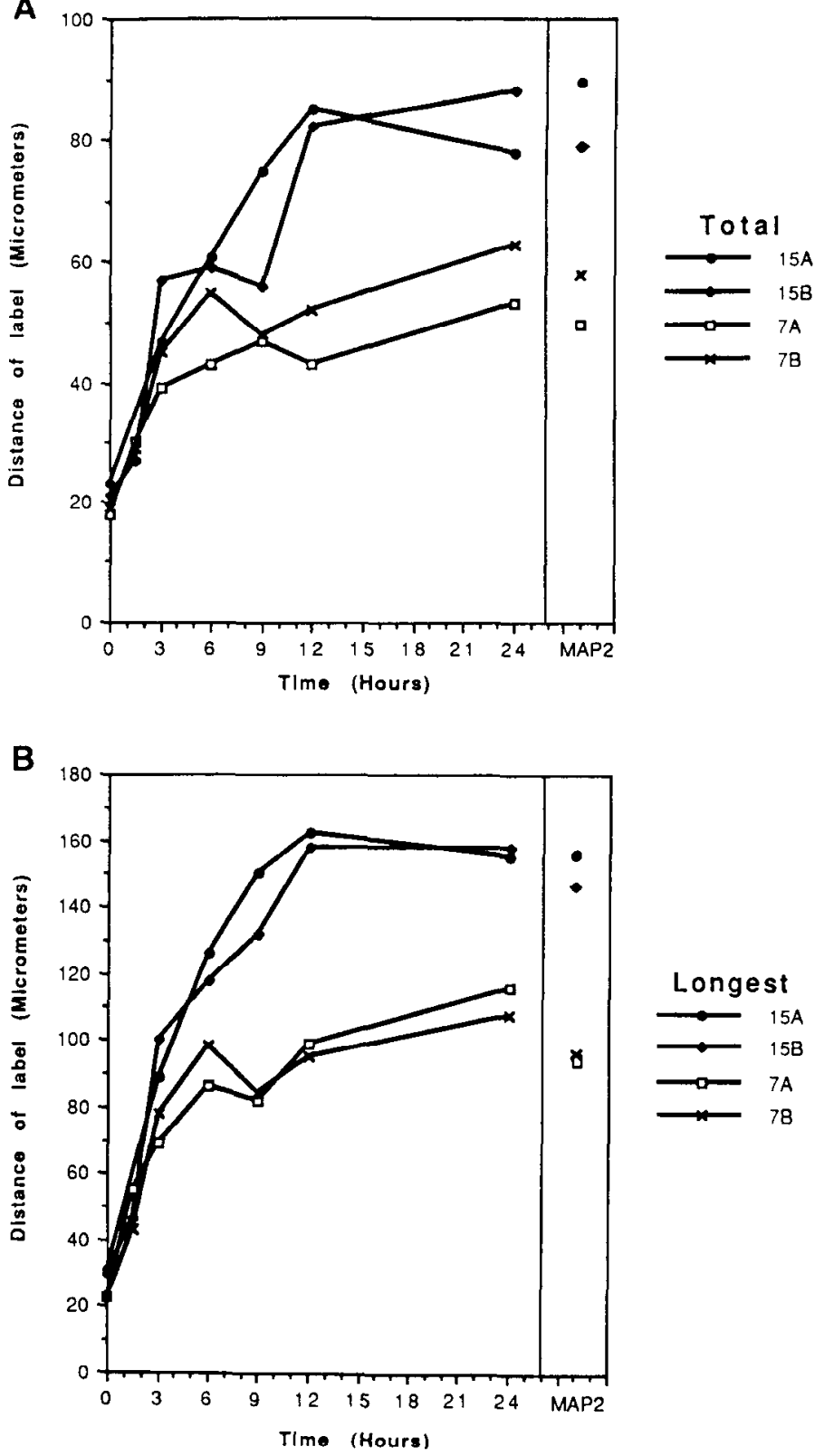

Figure 3. The time course of transport of ${ }^{3} \mathrm{H}-\mathrm{RNA}$. These graphs illustrate the time course of the increase in the mean distance of labeling at each chase interval in the total population of dendrites $(A)$ and the dendrites with the longest distance of label $(B)$ in all of the experiments. The individual data points to the right of the vertical line denote the mean dendritic length measured in MAP2-stained sister cultures. Error bars were not drawn because the standard deviation was large (roughly $50 \%$ of the mean), reflecting the variability inherent in the lengths of the dendrites themselves. The standard error of the mean ranged from 2 or $3 \mu \mathrm{m}$ (chase intervals, $1.5 \mathrm{hr}$ and longer) to 4 or $5 \mu \mathrm{m}$ (chase intervals, $0-1.5 \mathrm{hr}$ ).

By $6 \mathrm{hr}$ (experiment 7B) or $9 \mathrm{hr}$ (7A), there was no significant difference between the average distance of labeling and averagc dendritic length, indicating that labeling had reached the tips of the dendrites. In the 7-d-old cultures, the average distance of dendritic labeling at 12 and $24 \mathrm{hr}$ was slightly longer than the average dendritic length, estimated from MAP2 staining. This is not surprising, because at this age, MAP2 staining may not 

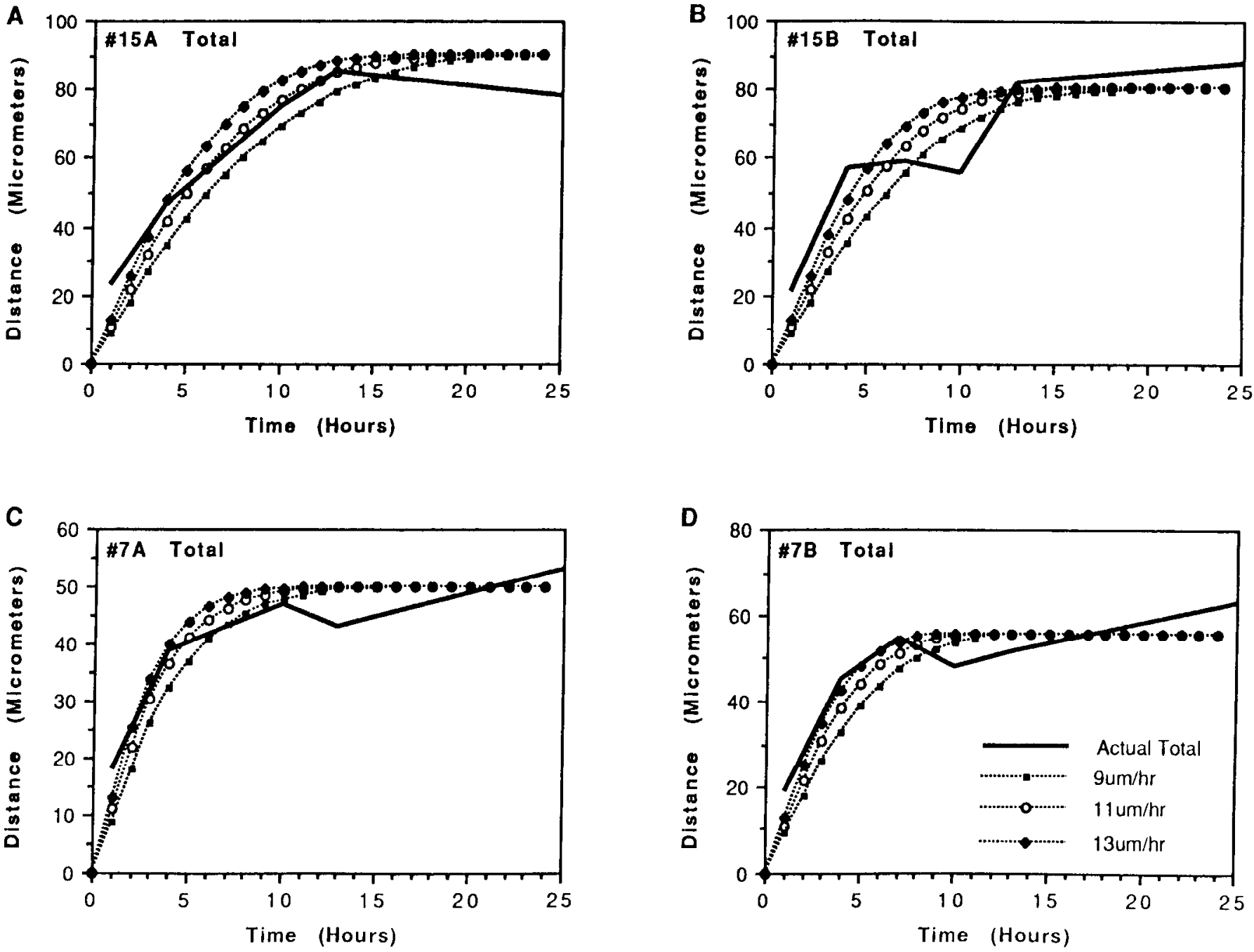

Figure 4. Predicted time course of increases in distance of labeling in total population of dendrites. Transport in the total population of dendrites was modeled using a constant velocity of 9,11 , or $13 \mu \mathrm{m} / \mathrm{hr}$. Least-square mean analyses revealed that transport was best fit when modeled at a velocity of $11 \mu \mathrm{m} / \mathrm{hr}$. In these comparisons, the curve of the observed velocity of transport was shifted $1 \mathrm{hr}$ to the right because the 0 -hr chase interval is actually $1 \mathrm{hr}$ after the beginning of the pulse labeling with ${ }^{3} \mathrm{H}$-uridine (bold line). $A$ and $B$, The predicted time course of the increases in the distance of labeling for the 2 experiments using 15 -d-old cultures, assuming transport velocities of 9,11, and $13 \mu \mathrm{m} / \mathrm{hr}$. $C$ and $D$, The predicted time course of the increases in the distance of labeling for the 2 experiments using 7-d-old cultures.

extend to the tips of dendritic growth cones (e.g., Goslin et al., 1988).

\section{The velocity of dendritic transport}

Velocity of transport (i.e., the displacement of RNA as a function of time) was calculated from the experimental data by determining the differences in the average distance of labeling at different times. For this analysis, transport velocity was calculated only in 15-d-old neurons because the mean distance of labeling could be accurately measured for $6 \mathrm{hr}$ in these longer dendrites. Considering the total population of dendrites, the apparent velocity of transport between $0-6 \mathrm{hr}$ was about 0.25 $\mathrm{mm} / \mathrm{d}$. The apparent velocity of transport calculated from the population of dendrites with the longest distance of labeling was approximately $0.5 \mathrm{~mm} / \mathrm{d}$. In the 7 -d-old neurons, the apparent rates of transport between $0-3 \mathrm{hr}$ were approximately the same as for the 15-d-old neurons for both the total population of dendrites and the dendrites exhibiting the longest distance of labeling (Fig. 3).

\section{Mathematical modeling of the time course of transport}

As noted in the introductory remarks, the differences in the apparent transport velocity between the total population of dendrites and the dendrites with the longest distance of labeling could be a result of the fact that the total population includes some relatively short dendrites. Alternatively, the differences could reflect an actual difference in transport velocity in dendrites of different lengths. Similarly, it is difficult to make a direct comparison of the time course of transport in 7- and 15$\mathrm{d}$-old neurons because the average length of the dendrites is so different between the 2 populations. Therefore, we developed a mathematical model that calculates the expected mean distance of transport at any transport interval in a population of cells of known dendritic lengths, assuming different velocities of transport. The calculations take into account the fact that there can be no further increase in the distance of labeling of a given dendrite once labeling has reached its tip.

The model makes possible the construction of theoretical 
A
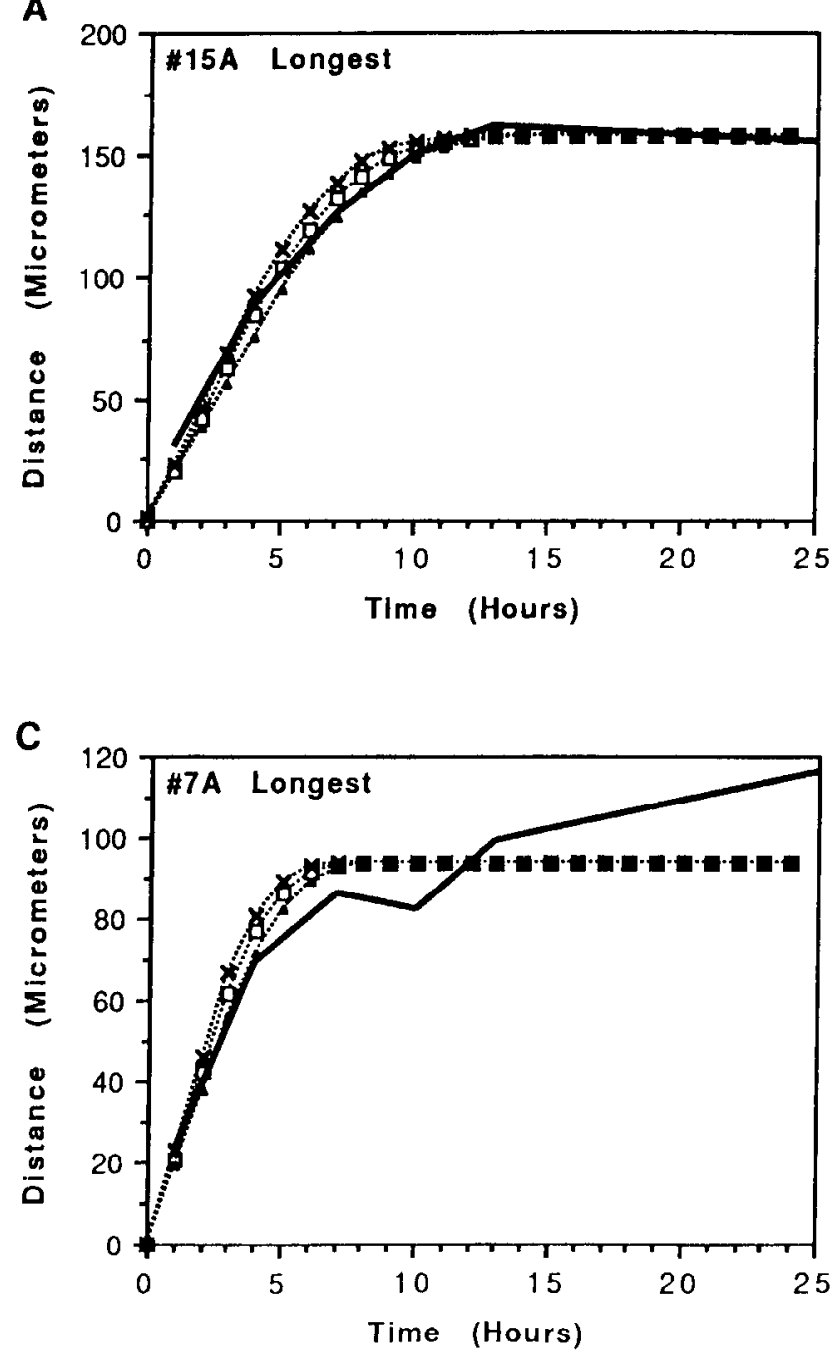

B

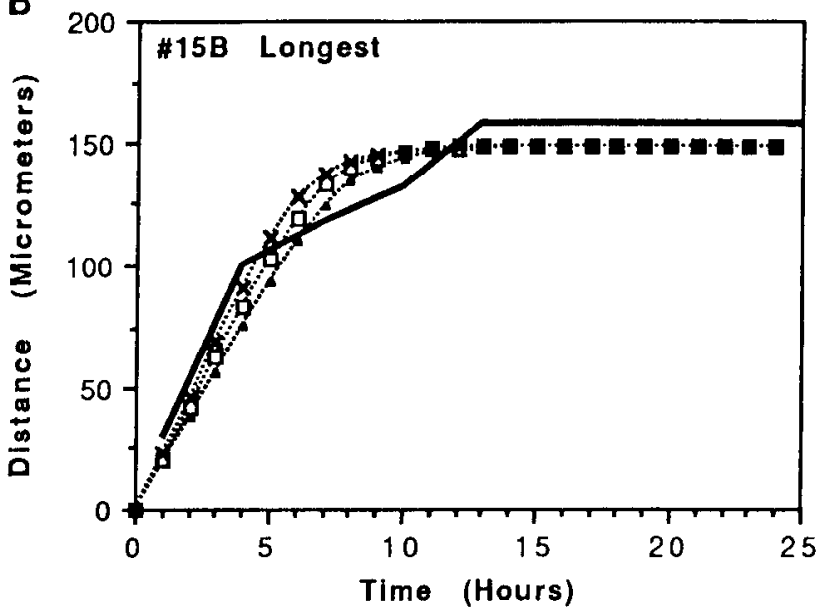

D

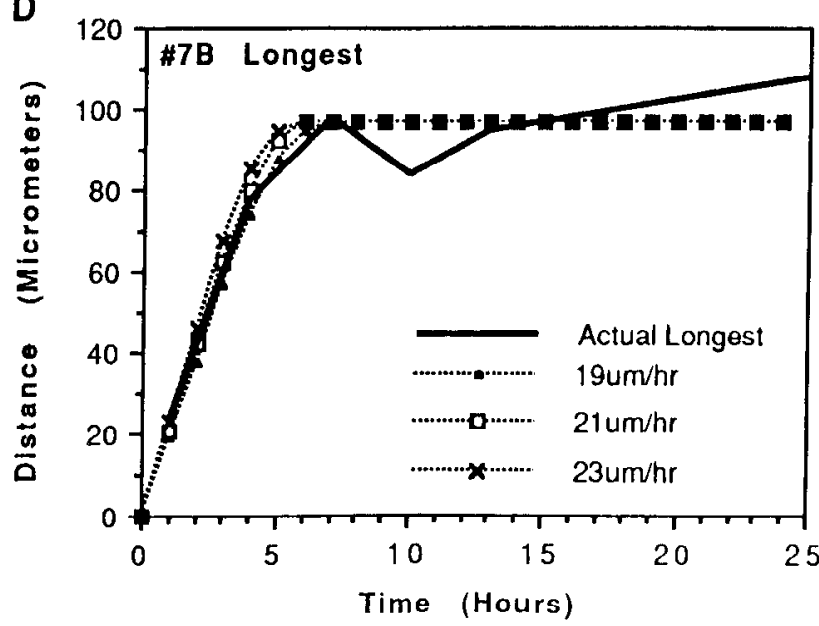

Figure 5. Predicted time course of increases in distance of labeling in longest dendrites. $A$ and $B$, The predicted time course of the increases in the distance of labeling for the 2 experiments using 15 -d-old cultures, assuming transport velocities of 19,21 , and $23 \mu \mathrm{m} / \mathrm{hr}$. $C$ and $D$, The predicted time course of the increases in the distance of labeling for the 2 experiments using 7-d-old cultures. The bold lines indicate the actual experimental values.

curves illustrating how the distribution of dendritic length affects measures of the increase in the distance of transport over time. For example, in an ideal system where (1) all dendrites are the same length, (2) the velocity of transport is constant throughout the length of the dendritc, and (3) the velocity of transport is the same in every dendrite, the distance of transport simply increases linearly until labeling reaches the ends of the dendrites. In contrast, in a population of processes of different lengths, the distance of transport increases linearly only until labeling reaches the ends of the shortest processes. Thereafter, the rate of increase in the distance of transport declines, leading to a negatively accelerating curve.

Comparison of the actual time course of the increase in labeling with models assuming various velocities of transport

The distribution of dendritic lengths for each experiment was evaluated using MAP2-stained sister cultures. These were considered appropriate because the distribution of labeling in the total population of dendrites after longer transport intervals (12 $\mathrm{hr}$ of transport in 15-d-old cultures, $9 \mathrm{hr}$ in 7-d-old cultures) was similar to the distribution of dendritic lengths based on MAP2 staining (see Table 2). The predicted time course of dendritic transport of RNA in the total population of dendrites was modeled assuming that the velocity of transport was constant and that the transport velocity was the same in all dendrites. This approach was used to analyze the data from each experiment at both ages. A least-squares analysis revealed that the best fit of the actual data from all experiments was obtained when the velocity of transport was assumed to be $11 \mu \mathrm{m} / \mathrm{hr}$ $(0.26 \mathrm{~mm} / \mathrm{d}$; see Fig. 4). This was true for both 7 - and 15 -d-old neurons.

As noted in our previous study, the apparent velocity of transport in the dendrites exhibiting the longest distance of labeling is greater than the apparent velocity in the total population of dendrites. If the actual velocity is underestimated because of the inclusion of short dendrites in the total population, then use of the model should correct for this error. Contrary to this prediction, when we modeled transport in the population of the longest dendrites of 25 neurons (defined from MAP2-stained sister cultures), the assumed velocity that produced a best-fitting 
Table 3. Mean distance of labeling in the total population of branched and unbranched dendrites

\begin{tabular}{|c|c|c|}
\hline \multirow[b]{2}{*}{ Experiment } & \multicolumn{2}{|c|}{ Mean distance of labeling $(\mu \mathrm{m}) \pm \mathrm{SD}$} \\
\hline & Unbranched & Branched \\
\hline \multicolumn{3}{|c|}{$15 \mathrm{~A}, 15$ days in culture } \\
\hline $3 \mathrm{hr}$ & $\begin{array}{l}46.9 \pm 26 \\
124\end{array}$ & $\begin{array}{l}68.8 \pm 25^{* *} \\
26\end{array}$ \\
\hline $6 \mathrm{hr}$ & $\begin{array}{l}53.2 \pm 33 \\
123\end{array}$ & $\begin{array}{l}93.2 \pm 39^{* *} \\
39\end{array}$ \\
\hline MAP2 & $\begin{array}{l}60.4 \pm 35 \\
86\end{array}$ & $\begin{array}{l}105.6 \pm 49^{* *} \\
194\end{array}$ \\
\hline \multicolumn{3}{|c|}{$15 \mathrm{~B}, 15$ days in culture } \\
\hline $1.5 \mathrm{hr}$ & $\begin{array}{l}33.6 \pm 11 \\
91\end{array}$ & $\begin{array}{c}44.2 \pm 13^{*} \\
9\end{array}$ \\
\hline $3 \mathrm{hr}$ & $\begin{array}{l}48.7 \pm 23 \\
117\end{array}$ & $\begin{array}{l}81.8 \pm 31^{* *} \\
55\end{array}$ \\
\hline $6 \mathrm{hr}$ & $\begin{array}{l}51.4 \pm 24 \\
155\end{array}$ & $\begin{array}{l}87.6 \pm 36^{* *} \\
60\end{array}$ \\
\hline MAP2 & $\begin{array}{l}64.8 \pm 33 \\
107\end{array}$ & $\begin{array}{l}88.9 \pm 39 * * \\
259\end{array}$ \\
\hline \multicolumn{3}{|c|}{$7 \mathrm{~A}, 7$ days in culture } \\
\hline $1.5 \mathrm{hr}$ & $\begin{array}{l}33.1 \pm 14 \\
103\end{array}$ & $\begin{array}{l}50.1 \pm 18^{* *} \\
29\end{array}$ \\
\hline $3 \mathrm{hr}$ & $\begin{array}{l}37.3 \pm 18 \\
115\end{array}$ & $\begin{array}{l}63.0 \pm 33^{* *} \\
32\end{array}$ \\
\hline MAP2 & $\begin{array}{l}42.2 \pm 21 \\
140\end{array}$ & $\begin{array}{l}63.3 \pm 30^{* *} \\
109\end{array}$ \\
\hline \multicolumn{3}{|c|}{$7 \mathrm{~B}, 7$ days in culture } \\
\hline $1.5 \mathrm{hr}$ & $\begin{array}{l}29.9 \pm 7 \\
83\end{array}$ & $\begin{array}{l}41.7 \pm 13^{*} \\
11\end{array}$ \\
\hline $3 \mathrm{hr}$ & $\begin{array}{l}44.2 \pm 19 \\
87\end{array}$ & $\begin{array}{l}61.9 \pm 21^{*} \\
20\end{array}$ \\
\hline MAP2 & $\begin{array}{l}53.5 \pm 26 \\
63\end{array}$ & $\begin{array}{l}67.2 \pm 25^{* *} \\
57\end{array}$ \\
\hline
\end{tabular}

The difference in the distances of labeling in branched and unbranched dendrites at each transport interval (or lengths as shown by MAP2 staining) was analyzed by tests of least-square mean ranks ( $t$ statistics). The sample size for each group is listed below the mean.

$* p<0.01$.

$* * p<0.0001$

curve was $21 \mu \mathrm{m} / \mathrm{hr}$, or $0.50 \mathrm{~mm} / \mathrm{d}$ (see Fig. 5). Analyses of the data from 7-d-old neurons yielded similar estimates of the most likely velucities for transport within the total population of dendrites and the dendrites exhibiting the longest distance of labeling. These results clearly reveal that differences in the distribution of the dendritic lengths between the total population and the population of longest dendrites cannot account for the observed differences in the time course of the increases in the distance of labeling.

\section{Modification of the model}

If the velocity of transport is faster in the longest dendrites, then one of the central assumptions underlying the best-fit estimates of the velocity is incorrect (that the velocity of transport is similar in all dendrites). Therefore, it was important to evaluate how differences in the velocity of transport in dendrites of different lengths would affect our estimates of the actual average velocity.
To evaluate this question, we generated theoretical transport curves assuming a set of velocities that varied systematically as a function of dendritic length using the actual population of dendrites from experiment $15 \mathrm{~A}$. We assumed a velocity of 21 $\mu \mathrm{m} / \mathrm{hr}$ in the longest dendrites. We then divided the total population of dendrites into the shortest, middle, and longest thirds. Assuming that $21 \mu \mathrm{m} / \mathrm{hr}$ would be the maximal velocity in the very longest dendrites, we assigned rates of $15 \mu \mathrm{m} / \mathrm{hr}$ or $18 \mu \mathrm{m} /$ $\mathrm{hr}$ to the longest third in different modeling situations, then calculated a series of transport curves assuming different velocities in the shortest and midlength dendrites (data not shown). When the velocity in the longest dendrite was set at $18 \mu \mathrm{m} / \mathrm{hr}$, a curve approximating the experimental curve was obtained when velocities of $3 \mu \mathrm{m} / \mathrm{hr}$ and $6 \mu \mathrm{m} / \mathrm{hr}$ were assigned to the shortest and midlength dendrites, respectively. A very similar curve was obtained assuming velocities of 3,9 , and $15 \mu \mathrm{m} / \mathrm{hr}$ (data not shown). The overall average velocity of transport in each of these conditions was $9 \mu \mathrm{m} / \mathrm{hr}$. Further modeling demonstrated that, though there were slight differences, an approximately equally good fit to the actual transport data was obtained by using any combination of velocities in which the mean velocity was $9 \mu \mathrm{m} / \mathrm{hr}$. Obviously, these calculations are based upon specific assumptions that cannot be verified. However, they offer some assurance that estimates of transport velocity based on this model are not grossly inaccurate.

\section{Comparison of dendritic transport in branched and unbranched dendrites}

\section{The velocity of transport in branched and unbranched dendrites}

The analyses presented above suggest that transport velocity may differ among the dendrites of a single neuron, depending on dendritic length. To investigate further possible differences in the velocity of RNA transport, we compared the time course of transport in branched versus unbranched dendrites. While this comparison is conceptually straightforward, several difficulties were encountered in distinguishing between branched and unbranched dendrites. One problem is that branched dendrites are, in general, longer than unbranched dendrites. Therefore, at long chase intervals, the distance of labeling in branched dendrites would be longer than in unbranched dendrites, independent of possible differences in transport rate. For this reason, we restricted our analysis to early intervals, before the transported material reaches the tips of the dendrites (6 hr or less in 15-d-old cultures, and $3 \mathrm{hr}$ or less in 7-d-old cultures). Howcver, at short chase intervals, when dendrites are labeled only over the most proximal segments, it is not possible to identify dendrites that branch distally. Examining the neurons using phase-contrast optics was not adequate because of the large number of axons that intersected the dendrites. Therefore, some of the dendrites classified as unbranched at the early intervals probably were, in fact, branched.

Table 3 compares the average distance of labeling in branched and unbranched dendrites at each chase interval. A least-square mean-rank analysis revealed that the distance of labeling in branched dendrites was significantly greater than in unbranched dendrites at every chase interval in all experiments $(p<0.01$; see Table 3). In fact, labeling appeared to reach the ends of branched dendrites sooner, even though they were longer than unbranched dendrites. These data suggest that the rate of RNA transport is faster in branched dendrites. Because some branched 

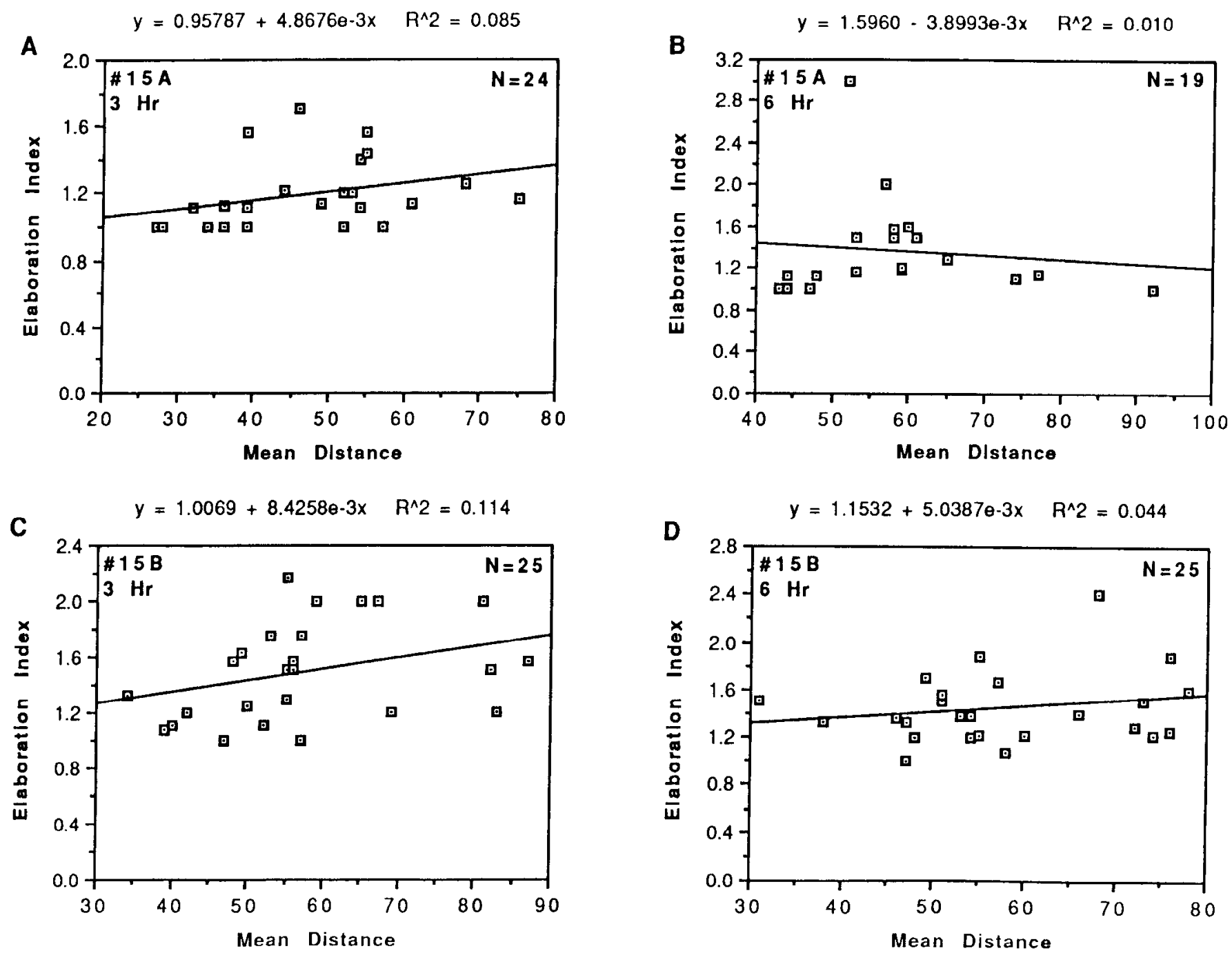

Figure 6. Regression analyses comparing EI and mean distance of labeling in all dendrites of each neuron. Neurons were only included in these analyses if no dendrites had been excluded (see Materials and Methods, Table 1). The number of neurons analyzed $(N)$ is listed in the upper right hand corner of each graph. Pearson's $r$ was calculated and transformed to a $z$ score. The level of significance of correlation was determined by calculating the $t$ statistic according to the formula, $t=r \sqrt{(n-2) /\left(1-r^{2}\right)}$.

dendrites were undoubtedly categorized as unbranched (see above), the difference in transport rate may even be greater than the data in Table 3 indicate.

It was of interest to determine whether transport velocity was faster in neurons with a greater proportion of branched dendrites. As a measure of the degree of dendritic branching, we calculated the elaboration index (EI) for each neuron, defined as the number of labeled dendritic tips divided by the number of primary dendrites. Regression analyses revealed that neurons with a greater El did not exhibit faster transport rates $(p>0.05$; see Fig. 6).

\section{Comparison of grain density over branched and unbranched dendrites}

Longer dendrites are also larger in diameter at their origins (Banker and Waxman, 1988). Therefore, there might be differences in the amount of the label entering different dendrites, which could influence measurements of transport. To examine this possibility, we compared the grain density over branched and unbranched dendrites. For this purpose, we evaluated grain density at different distances from the soma at 3,6 , and $12 \mathrm{hr}$ after labeling. Representative data are presented in Table 4. In general, grain density tended to be higher in branched dendrites. The differences were significant over proximal segments after $12 \mathrm{hr}$ of labeling ( $p \leq 0.04$ ). Differences were not significant at earlier time points $(p>0.05)$.

Modeling the increases in the distance of labeling over timc assuming diffusion

We have previously reported that much of the ${ }^{3} \mathrm{H}-\mathrm{RNA}$ in dendrites is bound to the cytoskeleton and that the migration of material into dendrites is inhibited by metabolic poisons. These observations argue against the possibility that the migration of RNA into dendrites occurs via diffusion (Davis et al., 1987). Nevertheless, mathematical modeling provides an alternative approach to assess whether the time course of dendritic labeling is consistent with migration of RNA by diffusion.

For these analyses, we assumed that diffusion would occur according to Einstein's relation for Brownian motion: $x^{2}=2 D t$, 

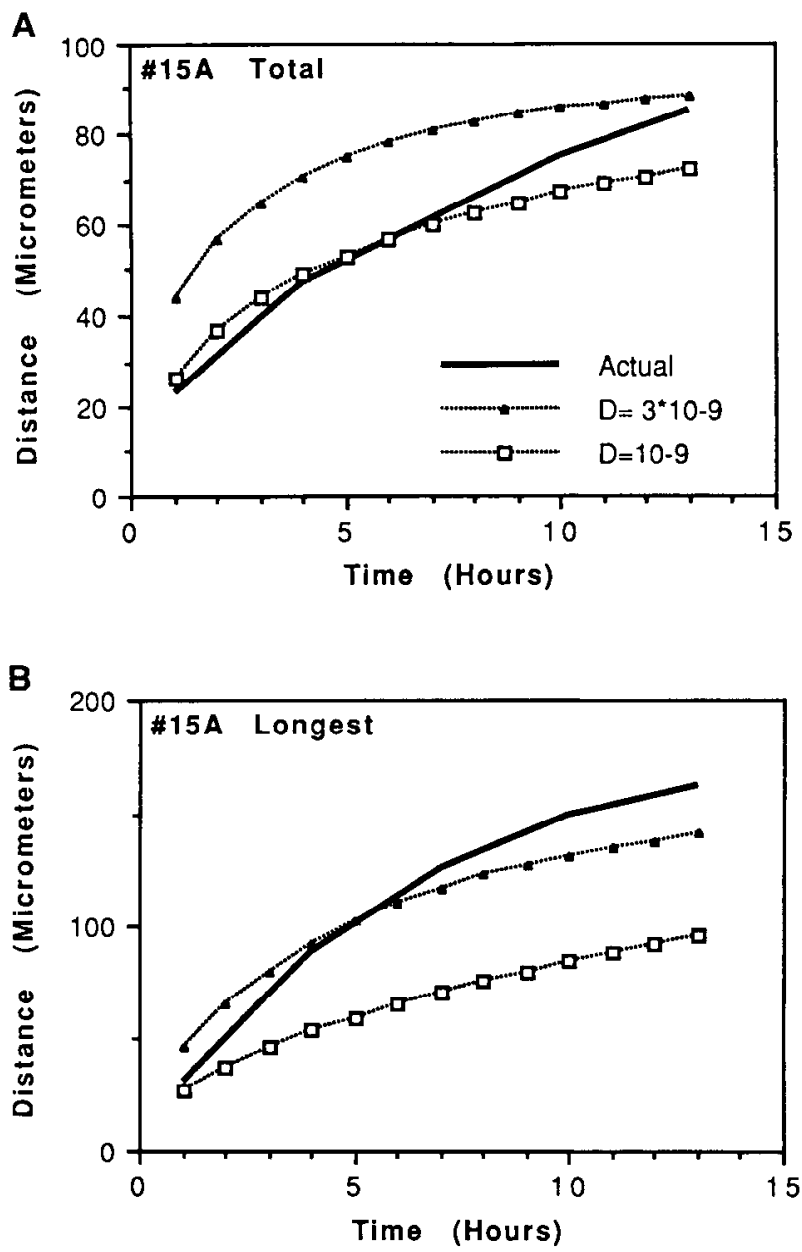

Figure 7. Predicted time course of increase in distance of labeling assuming diffusion. Diffusion was modeled using Einstein's relation, $x^{2}$ $=2 D t$, where $D=10^{-9} \mathrm{~cm}^{2} / \mathrm{sec}$ (open squares) or $3 \times 10^{-9} \mathrm{~cm}^{2} / \mathrm{sec}$ (solid triangles). The actual time course of transport in experiment $15 \mathrm{~A}$ is represented by a bold line. A. Comparison of the time course of transport in the total population of dendrites with diffusion modeled in the total population of dendrites. $B$, Comparison of the time course of transport in the dendrites with the longest distances of labeling with diffusion modeled in the longest dendrites.

where $x=$ distance, $D=$ the diffusion coefficient, and $t=$ time (Einstein, 1908, 1956). Using this equation, we calculated the time course of the increases in the distance of transport assuming different diffusion coefficients. As illustrated in Figure $7 A$, the predicted curves for diffusion do not resemble the experimental curves. The best fitting curve was obtained assuming $D=10^{-9}$ $\mathrm{cm}^{2} / \mathrm{sec}$. Theoretical curves that were generated to model transport appeared qualitatively more like the experimental curves. However, because of variability in the measurements of the distance of labeling, comparisons of the goodness of fit of the curves based on transport or diffusion were not significantly different (sum of squares $=579$, diffusion where $D=10^{-9} \mathrm{~cm}^{2} /$ sec; sum of squares $=327$, transport at $11 \mu \mathrm{m} / \mathrm{hr} ; F=1.77, p$ $=0.17$ ).

The short length of some of the dendrites in the total population makes it difficult to distinguish the kinetics of diffusion from those of transport. Both lead to negatively accelerating curves. Therefore, we also used the model to define the curve that would result from diffusion in the population of the longest dendrites. In this case, the best fit was obtained when $D=3 \times$
Table 4. Density of silver grains in branched and unbranched dendrites

\begin{tabular}{lclc}
$\begin{array}{llll}\text { Chase } \\
\text { interval }\end{array}$ & $\begin{array}{l}\text { Distance } \\
\text { from } \\
\text { nucleus }\end{array}$ & \multicolumn{3}{l}{ Mean density of silver grains } \\
\cline { 3 - 4 } $6 \mathrm{hr}$ & $25 \mu \mathrm{m}$ & $3.9 \pm 3.6(N=18)$ & $5.6 \pm 2.5(N=15)$ \\
& $50 \mu \mathrm{m}$ & $2.1 \pm 1.3(N=11)$ & $1.9 \pm 1.2(N-11)$ \\
& $75 \mu \mathrm{m}$ & $2.0 \pm 1.4(N=6)$ & $1.6 \pm 1.4(N=7)$ \\
& $100 \mu \mathrm{m}$ & $1.0 \pm 0.0(N=2)$ & $1.7 \pm 0.6(N=3)$ \\
$12 \mathrm{hr}$ & $25 \mu \mathrm{m}$ & $5.9 \pm 5.6(N=19)$ & $12.2 \pm 8.0(N=9)^{*}$ \\
& $50 \mu \mathrm{m}$ & $2.1 \pm 2.6(N=17)$ & $3.9 \pm 2.0(N=14)^{*}$ \\
& $75 \mu \mathrm{m}$ & $1.6 \pm 1.7(N=14)$ & $2.5 \pm 2.3(N=10)$ \\
& $100 \mu \mathrm{m}$ & $1.1 \pm 0.9(N=7)$ & $2.9 \pm 1.7(N=8)^{*}$ \\
& $125 \mu \mathrm{m}$ & $1.7 \pm 1.0(N-6)$ & $1.5 \pm 1.3(N-6)$ \\
& $150 \mu \mathrm{m}$ & - & $1.0 \pm 1.4(N=2)$ \\
& $175 \mu \mathrm{m}$ & - & $0.5 \pm 0.7(N=2)$
\end{tabular}

Grain density was measured in all of the labeled dendrites of 4 cells after 6 and $12 \mathrm{hr}$ of labeling in 15-d-old cultures (experiment 15A). Density is expressed as mean $\pm \mathrm{SD} . N=$ number of dendrites in the sample.

- No dendrites in sample.

$* p<0.05$

$10^{-9} \mathrm{~cm}^{2} / \mathrm{sec}$ (Fig. $7 B$ ). The model based on transport yields a significantly better approximation to the experimental curves (sum of squares $=442$ for transport model, 2531 for diffusion model; $F=5.73, p=0.005$ ).

\section{Discussion}

This study confirms and extends our previous evaluation of the dendritic transport of RNA (Davis ct al., 1987). Together, these studies provide the first characterization of the velocity of intracellular transport of RNA in any cell type. The present results suggest the following: (1) Recently synthesized RNA is transported at similar velocities in neurons at different stages of maturity, even though dendritic length and complexity differ substantially in neurons of different ages. (2) There are different transport velocities among the dendrites of a single neuron, with the fastest transport occurring in the longest dendrites. Although the longest dendrites tend to be branched and the distance of labeling is longer in branched dendrites, the overall average velocity of transport does not vary as a function of the degree of dendritic complexity. Taken together, these results suggest that dendritic transport is differentially regulated in the dendrites of an individual neuron. There are, however, a number of technical issues that must be considered in evaluating these conclusions. We will consider technical issues first, and then discuss the significance of our observations.

\section{Differences in dendritic transport between long and short dendrites of individual neurons}

Through the mathematical modeling approach, we were able to show that our previous assumption, that the best estimates of transport velocity would be obtained by focusing on the longest dendrites, was not correct. The underestimate of transport velocity as a result of the inclusion of short dendrites in the averages is not sufficient to account for the differences between the total population of dendrites and the dendrites exhibiting the longest distance of labeling. Instead, our results suggest that the velocity of dendritic transport varies as a function of dendritic length, with the longest dendrites showing the highest velocities of transport.

With regard to the calculations of the differential velocity of 
transport in long dendrites, it is important to emphasize that our measures focused on the single dendrite of each neuron that exhibited the longest distance of labeling. This may not constitute the longest dendrite in every case. However, qualitative observations suggest that the dendrites exhibiting the longest distances of labeling are at least among the 1-2 longest dendrites of the neuron. A precise resolution of this issue will require direct comparisons of the distance of labeling and dendritic length using a combination of autoradiography and MAP2 immunocytochemistry on individual neurons.

One other complicating factor that must be considered is that labeling over longer dendrites may be higher because these dendrites have a larger diameter. If the labeling is greater, then the apparent distance of transport may be greater in longer dendrites simply because the labeling may be above threshold for detection for a greater distance. In fact, dendritic diameter at the junction of the soma and dendrite is proportional to dendritic length (Banker and Waxman, 1988). Therefore, our calculations of the distance of transport in the proximal dendritic segment may be in error (i.e., at early chase intervals). However, because the terminal diameter of all dendrites is similar (Banker and Waxman, 1988), measurements of the distance of labeling in distal dendritic regions should not be biased by this factor. Therefore, this potential complicating factor is not likely to affect the central conclusion that the velocity of transport is faster in the longest dendrites.

\section{Comparisons of transport in branched and unbranched dendrites}

Other issues arise in comparing transport in branched and unbranched dendrites. One major problem is that the identification of branched and unbranched dendrites is flawed, as detailed in the Results. However, errors introduced by the inclusion of branched dendrites in the unbranched sample would only tend to reduce the differences between the 2 populations. Therefore, the basic conclusion, that transport is faster in branched dendrites, is probably sound; moreover, the extent of the difference in transport velocity might actually be greater than the data suggest.

Another problem related to the comparison of branched and unbranched dendrites is that branched dendrites also tend to be longer. Therefore, the velocity of transport in branched dendrites may be faster because they are longer, not because of some difference that is related to branching per se. Whatever the reason, the results do suggest that neurons transport material at different velocities into individual dendrites of different morphologies.

While the velocity of transport differs when comparing the branched and unbranched dendrites of individual neurons, the average velocity of transport is not apparently faster in neurons with a greater proportion of branched dendrites. Again, this result is consistent with the conclusion that the average velocity of transport in different types of neurons is comparable and that differences in transport are expressed as differences in transport velocities among individual dendrites.

\section{Summary of differential transport velocities}

Two aspects of the data suggest that RNA is transported at different velocities in different dendrites of an individual neuron: (1) Recently synthesized RNA is apparently transported more rapidly into the longest dendrites and more slowly into the shortest dendrites of both 7-and 15-d-old neurons. (2) RNA is apparently transported more rapidly into branched dendrites than it is into unbranched dendrites of the same neuron. Although the velocity of transport may vary considerably between different dendrites, the mean transport velocity is apparently similar regardless of dendritic complexity.

Differential transport velocities may not be uncommon in slow transport systems. For example, in winter flounder, which possess optic nerves that are naturally asymmetrical in length, Murray (1974) demonstrated that the velocity of migration of slowly transported proteins is faster in the longer optic nerve. Taken together, these results suggest that RNA transport may be selectively regulated in individual dendrites on the basis of length or some other aspect of dendritic morphology or physiology.

\section{Summary of transport velocity in neurons of different ages}

Because the velocity of transport is faster in longer dendrites, the overall average velocity of transport is probably different than predicted by the simple model. However, the errors introduced by having multiple velocities would be similar in younger and older neurons. Because the velocity predicted by the model is the same in the 2 populations, it is likely that the actual average velocity of transport is also comparable in 7- and 15-d-old neurons. The absence of any apparent differences in dendritic transport in cultures of these ages suggests that there is a constant requirement for recently synthesized RNA throughout a relatively broad range of development.

Our results reveal that the velocity of transport in the longest dendrites of 7-d-old neurons is comparable to the velocity of transport in the longest dendrites of 15-d-old neurons, despite 2 -fold differences in absolute length. One interesting interpretation of these results is that the longest and most elaborate dendrites of 7-d-old neurons may be the ones that are destined to grow to be the longest and most elaborate dendrites in mature neurons. If this is true, a determination of the complexity of individual dendrites may be made relatively early in the developmental history of the neuron and may be related to the transport machinery that is present in those dendrites.

\section{Significance}

Taken together, the present results suggest that individual neurons regulate dendritic transport of recently synthesized RNA on a dendrite-by-dendrite basis. How this regulation occurs is currently a matter for speculation. Whatever the mechanism, our studies of dendritic transport suggest that the intracellular transport mechanisms of neurons may be considerably more complex than previously suspected. In fact, it seems possible that neurons may possess as many different mechanisms for dendritic transport as are available for axonal transport. Delineation of the properties of these transport mechanisms will certainly provide new insights into the cellular mechanisms underlying neuronal operation.

Based on our studies of RNA transport, we have hypothesized that mRNAs may be differentially distributed within neurons and that this may contribute to the establishment of a mosaic postsynaptic receptor surface (Davis et al., 1987; Steward et al., 1988; sec also Gordon-Wccks, 1988). It has now been demonstrated directly that only a restricted population of messages are present within dendrites (Levinthal et al., 1987; Garner et al., 1988; Papandrikopoulou et al., 1989). For example, the mRNA encoding the high-molecular-weight form of MAP2, a protein that is restricted to the somatodendritic domain, is present in dendrites and somata. Messengers for several other cytoskeletal proteins that are not selectively distributed are largely 
restricted to the cell body. Moreover, in the case of muscle cells, it has been demonstrated that the differential distribution of mRNAs contributes to the selective distribution of both cytoplasmic and cell-surface proteins (Pavlath et al., 1989; Ralston and Hall, 1989). These findings underscore the importance of intracellular transport and sorting of RNA and the need to elucidate the cellular basis of transport and the molecular signals underlying its selectivity.

Note added in proof: Since this manuscript was accepted for publication, a report has appeared that provides additional information about which mRNAs might be present in dendrites. Using in situ hybridization, the researchers showed that the mRNA for the alpha subunit of $\mathrm{Ca}^{2} / \mathrm{cal}^{-}$ modulin-dependent protein kinase was present in high concentrations in neuropil zones that contain few neuronal cell bodies but large numbers of dendrites-i.e., the molecular layers within the hippocampus and layer I of the cerebral cortex (Burgin et al., 1990). Interestingly, the mRNA for the beta subunit of the kinase appeared to be restricted to neuronal cell bodies.

In addition, another report appeared that provides an estimate of the rate of mRNA transport in Xenopus oocytes (Yisraeli et al., 1990). By measuring the rate of translocation of maternal mRNAs that are differentially distributed within oocytes, the researchers estimated that the rate of transport is on the order of $100 \mu \mathrm{m}$ per day. This study also revealed that agents that disrupt microtubules (nocodazole and colchicine) disrupt mRNA translocation, whereas agents that disrupt microfilaments (cytochalasin B) lead to a uniform distribution of mRNA, suggesting a disruption of an annchoring mechanism. These results are consistent with our previous studies in neurons (Davis et al., 1987), suggesting that transport of RNA occurs as a result of binding to the cytoskeleton.

\section{References}

Banker GA, Cowan WM (1979) Further observations on hippocampal neurons in dispersed cell culture. J Comp Neurol 187:469-494.

Banker GA, Waxman AB (1988) Hippocampal neurons generate natural shapes in cell culture. In: Intrinsic determinants of neuronal form and function (Lasek R, Black M, eds), pp 61-82. New York: Liss.

Bartlett WP, Banker GA (1984a) An electron microscopic study of the development of axons and dendrites by hippocampal neurons in culture. I. Cells which develop without intercellular contacts. J Neurosci 4:1944-1953.

Bartlett WP, Banker GA (1984b) An electron microscopic study of the development of axons and dendrites by hippocampal neurons in culture. II. Synaptic relationships. J Neurosci 4:1954-1965.

Binder LI, Frankfurter A, Rebhun LI (1986) Differential localization of MAP-2 and tau in mammalian neurons in situ. Ann NY Acad Sci 466:145-166.

Black MM, Lasek RJ (1977) The presence of transfer RNA in the axoplasm of the squid giant axon. J Neurobiol 8:229-237.

Bottenstein JE, Sato GH (1979) Growth of a rat neuroblastoma cell line in serum-free supplemented medium. Proc Natl Acad Sci USA 76:514-517.

Burgin KE, Waxham MN, Rickling S, Westgate SA, Mobley WC, Kelly PT (1990) In situ hybridization histochemistry of $\mathrm{Ca}^{2+} /$ calmodulindependent protein kinase in developing rat brain. $\mathrm{J}$ Neurosci 10:17881798.

Caceres A, Binder LI, Payne MR, Rebhun L, Steward O (1984b) Differential subcellular localization of tubulin and the microtubule associated protein MAP2 in brain tissue as revealed by immunocyto- chemistry with monoclonal hybridoma antibodies. J Neurosci 4:394410.

Caceres A, Banker GA, Binder LI (1986) Immunocytochemical localization of tubulin and microtubule-associated protein 2 during the development of hippocampal neurons in culture. J Neurosci 6:714722 .

Davis L, Banker GA, Steward O (1987) Selective dendritic transport of RNA in hippocampal neurons in culture. Nature 330:477-479.

Einstein A (1908) Elementare theorie der Brownschen Bewegung. Z Elektrochem Angew Phys Chem 14:235-239.

Einstein A (1956) Investigation on the theory of the Brownian movement. New York: Dover.

Garner CC, Tucker RP, Matus A (1988) Selective localization of messenger RNA for cytoskeletal protein MAP2 in dendrites. Nature 336: 674-677.

Gordon-Weeks P (1988) RNA transport in dendrites. TINS 11:342343.

Goslin K, Banker G (1990) Rat hippocampal neurons in low density culture. In: Culturing nerve cells (Banker G, Goslin K, eds). Cambridge, MA: MIT (in press).

Goslin K, Schreyer DJ, Skene JHP, Banker GA (1988) Development of neuronal polarity: GAP-43 distinguishes axonal from dendritic growth cones. Nature 336:672-674.

Levinthal F, Oberdick J, Yang SM, Levinthal C (1987) Specific mRNA identified during development in mouse Purkinje cells and their dendrites. Soc Neurosci Abstr 13:1708.

Matus A, Bernhardt R, Hugh-Jones T (1981) High molecular weight microtubule-associated proteins are preferentially associated with dendritic microtubules in brain. Proc Natl Acad Sci USA 78:30103014.

Murray M (1974) Axonal transport in the asymmetric optic axons of flatfish. Exp Neurol 42:636-646.

Papandrikopoulou A, Doll T, Tucker RP, Garner CC, Matus A (1989) Embryonic MAP2 lacks the cross-linking sidearm sequences and dendritic targeting signal of adult MAP2. Nature 340:650-652.

Pavlath GK, Rich K, Webster SG, Blau HM (1989) Localization of muscle gene products in nuclear domains. Nature 337:570-573.

Ralston E, Hall ZW (1989) Transfer of a protein encoded by a single nucleus to nearby nuclei in multinucleated myotubes. Science 244 : $1066-1069$.

Steward O (1983) Alterations in polyribosomes associated with dendritic spines during the reinnervation of the dentate gyrus of the adult rat. J Neurosci 3:177-188.

Steward O, Falk PM (1986) Protein synthetic machinery at postsynaptic sites during synaptogenesis: a quantitative study of the association between polyribosomes and developing synapses. J Neurosci 6 : $412-423$.

Steward O, Levy WB (1982) Preferential localization of polyribosomes under the base of dendritic spines in granule cells of the dentate gyrus. J Neurosci 2:284-291

Steward O, Davis L, Dotti C, Phillips LL, Rao A, Banker GA (1988) Protein synthesis and processing in cytoplasmic microdomains beneath postsynaptic sites on CNS neurons: a mechanism for establishing and maintaining a mosaic postsynaptic receptive surface. $\mathrm{J}$ Mol Neurobiol 2:1-35.

Tucker RP, Garner CC, Matus A (1988) In situ localization of microtubule-associated protein mRNA in the developing and adult rat brain. Neuron 2:1245-1256.

Yisraeli JK, Sokol S, Melton DA (1990) A two-step model for the localization of maternal mRNA in Xenopus oocytes: involvement of microtubules and microfilaments in the translation and anchoring of Vg1 mRNA. Development 108:289-298.

Zanakis MF, Eskin B, Ingoglia NA (1984) Evidence that multiple species of aminoacylated transfer RNA are present in regenerating optic axons of goldfish. Neurochem Res 9:249-262. 